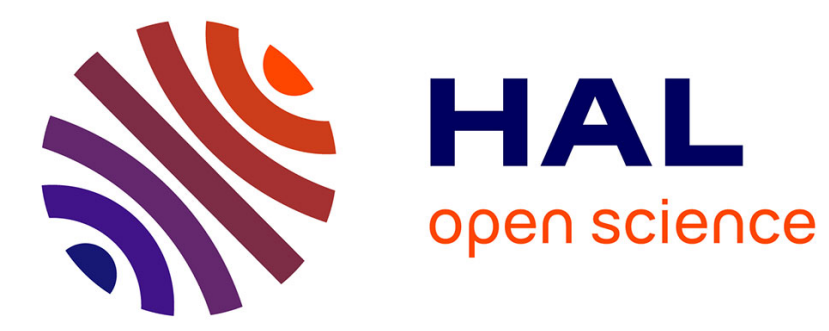

\title{
Total et le gazoduc birmano-thaïlandais
}

Eric Charmes

\section{To cite this version:}

Eric Charmes. Total et le gazoduc birmano-thaïlandais. Flux - Cahiers scientifiques internationaux Réseaux et territoires, 2007, 70, pp.59-64. halshs-00278341

\section{HAL Id: halshs-00278341 https://shs.hal.science/halshs-00278341}

Submitted on 12 May 2008

HAL is a multi-disciplinary open access archive for the deposit and dissemination of scientific research documents, whether they are published or not. The documents may come from teaching and research institutions in France or abroad, or from public or private research centers.
L'archive ouverte pluridisciplinaire HAL, est destinée au dépôt et à la diffusion de documents scientifiques de niveau recherche, publiés ou non, émanant des établissements d'enseignement et de recherche français ou étrangers, des laboratoires publics ou privés. 


\section{Total et le gazoduc birmano-thaïlandais}

Eric Charmes, Flux, rub. « Sens de l’événement », n70, octobre-décembre 2007, p. 59-64

Récemment, la Birmanie est revenue sur le devant de la scène internationale, avec d'importantes manifestations contre la junte militaire au pouvoir. A cette occasion, le groupe pétrolier français Total a, encore une fois, vu sa contribution à l'exploitation du gaz birman mise en question. La présence du groupe en Birmanie a même pour la première fois été critiquée officiellement par le chef de l'Etat français. Ce dernier a ainsi déclaré le 26 septembre 2007, à l'issue d'une rencontre avec l'opposition birmane : « La France appelle l'ensemble des sociétés privées, Total par exemple, à faire preuve de la plus grande retenue s'agissant des investissements en Birmanie, en demandant qu'il n’y en ait pas de nouveaux. »" Certes, Total n'envisage pas d'accroître sa présence en Birmanie et Nicolas Sarkozy n’a pas, à la différence de nombreuses organisations non gouvernementales, demandé un retrait du groupe pétrolier. Mais, compte tenu de l'actualité médiatique du moment, la mise en cause est forte.

L'arrivée officielle de Total en Birmanie remonte à 1992 avec la signature d'un contrat dit « de partage de production ». Depuis cette date, le groupe français pilote un consortium qui gère une plate-forme off-shore d'extraction de gaz (implantée sur le site de Yadana en mer d'Andaman) et un gazoduc qui relie ce site à la frontière birmano-thaïlandaise (la Thaïlande achète l'essentiel de la production et le groupe thaïlandais PTT contribue à hauteur de $25 \%$ au consortium piloté par Total). L’investissement réalisé représente plus d’un milliard de dollars, soit à ce jour l'investissement étranger le plus important en Birmanie ${ }^{2}$. Cette opération contribue incontestablement au développement économique de ce pays encore très pauvre. Mais la présence de Total apparaît à certaines organisations non gouvernementales comme un soutien à la junte militaire actuellement au pouvoir.

Une telle situation n'est pas exceptionnelle et sur la page d'accueil du site Internet dédié à la présentation de son action en Birmanie, le groupe constate, un peu fataliste : « La carte des gisements d'hydrocarbures dans le monde ne coïncide pas avec celle des régimes démocratiques. Aussi les groupes pétroliers sont-ils souvent interpellés par la société civile qui les interroge sur leurs méthodes de travail dans des pays difficiles, sur leurs rapports avec les pouvoirs en place, sur les mesures de sécurité visant à protéger leurs installations et sur l’emploi fait par les états hôtes de leurs recettes pétrolières. » Dans le cas de la Birmanie, les interrogations de la société civile sont d'autant plus pressantes que les Etats-Unis ont interdit à leurs entreprises d'investir dans ce pays et que de nombreuses sociétés occidentales ont fermé leurs filiales au cours des dernières années.

\footnotetext{
${ }^{1}$ Dépêche Reuters du 26 septembre 2007, reprise par de très nombreux journaux.

${ }^{2}$ Le nom officiel de la Birmanie est le Myanmar. Ce nom a été donné au pays par une junte militaire en 1989 alors qu'elle venait de prendre le pouvoir suite à un coup d’Etat. Nous utiliserons ici le nom de Birmanie comme le font la plupart des organisations qui ne reconnaissent pas la légitimité du pouvoir en place.
} 


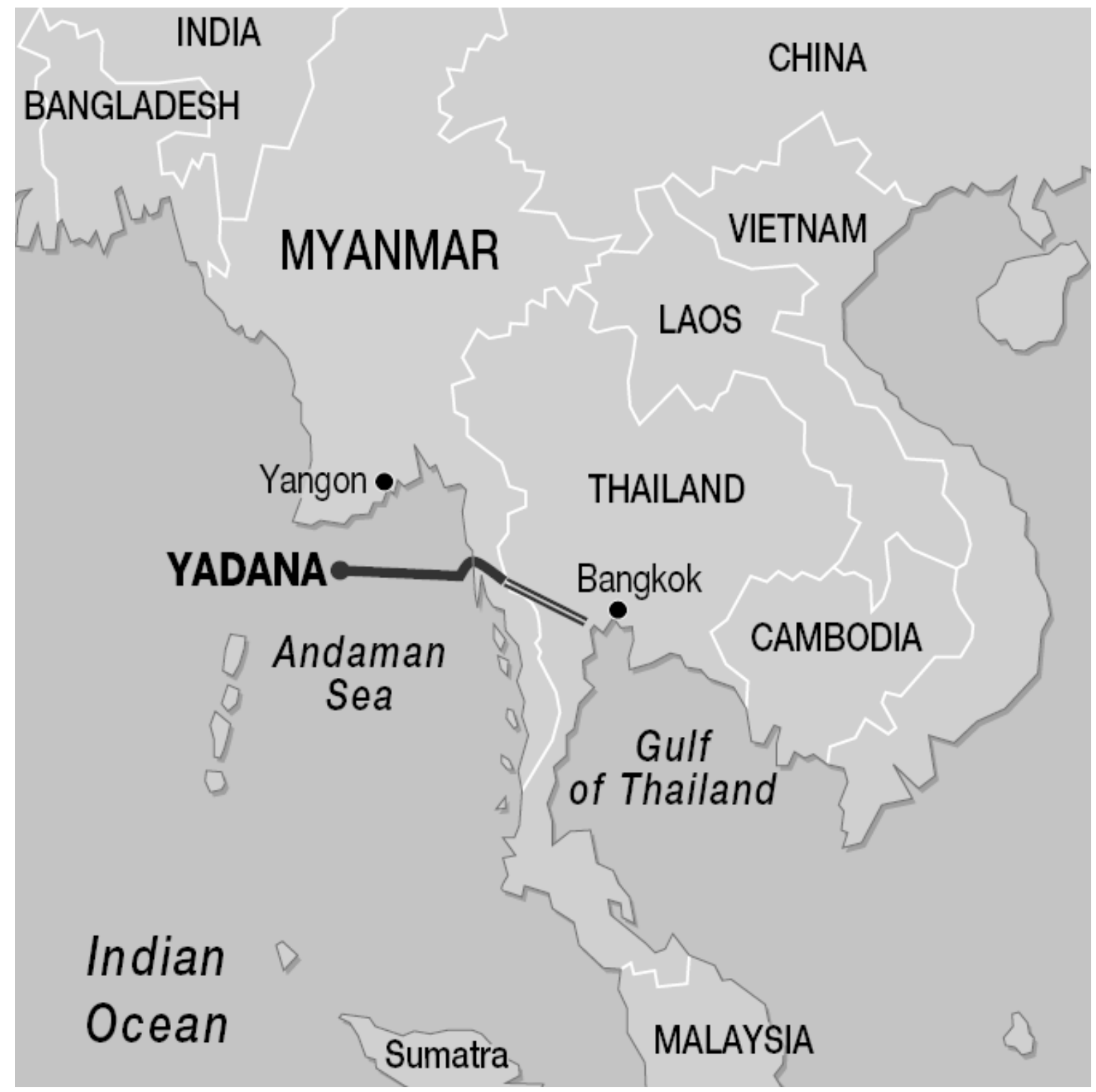

Le gisement et son gazoduc. La portion terrestre entre la mer d'Andaman et la frontière thaïlandaise a une longueur de 63 kilomètres (source : http://birmanie.total.com)

\section{« La firme française Total est devenue le principal soutien du régime militaire birman »}

Le moins que l'on puisse dire est que l'annonce officielle de l'investissement de Total en Birmanie est survenue à une date malvenue. La figure de proue de l'opposition démocratique, Aung San Suu Kyi, venait de recevoir le prix Nobel de la paix en 1991 pour sa lutte pacifique contre le pouvoir militaire en place. Par ailleurs, divers rapports mettaient en évidence un recours massif au travail forcé (longtemps considéré dans ce pays comme une sorte d'impôt légitime), des exécutions sommaires d'opposants politiques, un financement du pouvoir en place par le trafic de drogue (la Birmanie serait le premier producteur mondial d’héroïne), des 
exactions multiples contre les minorités ethniques du pays (notamment les Mon et les Karen), etc. La présence de Total a donc immédiatement suscité une opposition très forte.

Cette opposition a été alimentée par le fait qu’Aung San Suu Kyi s’est prononcée à plusieurs reprises contre les investissements étrangers en Birmanie, et plus particulièrement contre celui réalisé par Total. Elle a notamment déclaré au journal Le Monde en 1996 : « la firme française Total est devenue le principal soutien du régime militaire birman » ${ }^{3}$. Aung San Suu Kyi ne s'exprime pas qu'en son nom propre : son parti a remporté plus de $80 \%$ des sièges lors des élections nationales organisées par la junte militaire en 1990 et ensuite annulées par cette même junte. Pour beaucoup, Aung San Suu Kyi représente donc le pouvoir légitime dans ce pays. Son autorité est d'autant plus grande qu'elle a refusé un exil qui lui était proposé et a préféré rester en résidence surveillée, sacrifiant sa liberté pour mieux peser sur le régime.

Cette position vaut tous les encouragements pour ceux qui sont en faveur d'une mise au ban des pays totalitaires et qui estiment que les liens commerciaux avec ces derniers doivent être coupés. Condamner le quatrième groupe pétrolier mondial permet en outre de donner un écho particulier à la situation politique du pays. Elle permet d'agréger aux opposants au régime birman non seulement les organisations non gouvernementales (ONG) qui luttent pour la démocratie et les droits de l’homme, mais aussi les organisations alter-mondialistes.

De fait, la mobilisation est forte. Parmi les actions récentes, on peut citer un documentaire qui a commencé à être montré dans divers festivals en 2006. Réalisé par Milena Kaneva et intitulé « Total Denial », il a reçu à Prague un prix des mains de Vaclav Havel ${ }^{4}$. En 2005, une brochure de 63 pages intitulée « TOTAL ${ }^{5}$ pollue la démocratie. Stoppons le TOTALitarisme en Birmanie » a été mise en ligne sur le site de nombreuses organisations non gouvernementales $^{6}$. Elle est signée par Actions en Birmanie, Agir ici, la Fédération internationale des ligues des droits de l'homme (FIDH), France Liberté (fondation Danielle Mitterrand), Info Birmanie, Justice et solidarités mondiale (JSM) et Sud Chimie. La même année, la principale organisation d’opposition anglophone, Burma Campaign UK, a mis en ligne une brochure similaire intitulée « TOTALitarian Oil. Total Oil: Fuelling the Oppression in Burma $»^{7}$.

La condamnation qui ressort de la lecture de ces documents paraît sans appel. Trois accusations peuvent être distinguées : tout d'abord, la plate-forme offshore et le gazoduc financés par Total sont parmi les principales sources légales de devises pour la dictature birmane et sont à ce titre des facteurs cruciaux pour son maintien au pouvoir (la Birmanie retirerait du projet un revenu annuel estimé entre 200 et 450 millions de dollars, soit potentiellement $5 \%$ de son $\mathrm{PIB}^{8}$ ). Ensuite, la présence de Total en Birmanie empêcherait l'Union européenne de s'aligner sur la position très ferme prise par les Etats-Unis à l'égard de la Birmanie. De nombreux éléments attestent effectivement que la retenue de l’Europe est liée

\footnotetext{
${ }^{3}$ Edition du 20 juillet 1996, entretien avec Frédéric Bobin.

${ }^{4}$ www.totaldenialfilm.com

${ }^{5}$ Les majuscules sont des auteurs.

${ }^{6}$ voir, entre autres : www.fidh.org/IMG/pdf/mm04062005fr.pdf

${ }^{7}$ voir : www.burmacampaign.org.uk/total_report.html

${ }^{8}$ Le PIB de ce pays était d'environ 9 milliards de dollars en 2006 pour une population d'environ 55 millions d'habitants. Source : US Department of State.
} 
à la position française sur ce dossier. Ces deux premiers griefs sont toutefois d'ordre politique et n’engagent pas directement la responsabilité de Total. Ce n'est pas le cas de la troisième accusation qui met en cause des manquements au respect des droits de l'homme. De fait, le partenaire local de Total (MOGE, actionnaire à 15 \% de l'opération) utilise l'armée birmane pour garantir la sécurité du gazoduc. Or cette dernière se livre de manière routinière à des exactions dont certaines relèveraient du crime contre l'humanité (qualification proposée par l’Organisation internationale du travail pour la saisine de la cour pénale internationale concernant le recours au travail forcé par les autorités birmanes ${ }^{9}$ ).

\section{Des contestations portées sur le terrain juridique}

Comme fréquemment dans ce genre de situation, les contestations suscitées par la présence de Total en Birmanie se sont portées sur le terrain juridique. Des plaintes ont ainsi été déposées, accusant le groupe d'avoir (indirectement) recouru au travail forcé pour construire la section terrestre du gazoduc. L'une de ces plaintes a été déposée en France en 2002 par huit victimes birmanes. Le tribunal de grande instance de Nanterre a certes rendu une ordonnance de nonlieu en 2006, mais c’est après que Total a constitué en 2005 un fonds de solidarité de 5,2 millions d'euros destinés d'une part, à « offrir une compensation aux huit plaignants ainsi qu’à toute personne pouvant justifier s'être trouvée dans une situation semblable dans la région de construction du gazoduc et d'autre part, au financement d'actions humanitaires au profit de réfugiés birmans de la région ${ }^{10}$. La même année, le partenaire américain de Total dans cette affaire, Unocal, a réglé « par voie transactionnelle » un contentieux avec des ressortissants birmans qui avaient déposé des plaintes devant les tribunaux fédéraux américains et devant ceux de l'Etat de Californie ${ }^{11}$.

Total assure que les accusations dont il fait l'objet sont non fondées en fait comme en droit, et affirme avoir mis en place un fonds de compensation seulement pour des raisons humanitaires. Les organisations opposées à la présence de Total en Birmanie voient au contraire dans cette démarche la volonté de mettre un terme à une procédure embarrassante et un aveu de culpabilité : si l’entreprise avait été certaine de gagner le procès qui lui était intenté, elle n’aurait pas cherché un arrangement amiable hors des tribunaux.

\footnotetext{
${ }^{9}$ Voir le compte-rendu de la $297^{\mathrm{e}}$ session du conseil d'administration du bureau international du travail (Genève, novembre 2006), www.ilo.org

${ }^{10}$ Total, Total au Myanmar, un engagement durable, 2006, p. 40, brochure mise en ligne sur le site http://birmanie.total.com/index.htm

${ }^{11}$ Pour le détail de ce procès, voir www.earthrights.org
} 


\section{« Nos activités dans le cadre du projet Yadana contribuent au bien-être des populations du Myanmar »}

Face à ces diverses accusations, Total s'efforce de faire connaître les éléments qui lui sont favorables. Le groupe a ainsi créé un site Internet entièrement dédié à la justification de sa présence en Birmanie ${ }^{12}$. Ce site, assez complet, entend apporter des réponses à ceux qui s’interrogent. Les critiques des ONG sont abordées sans détour (notamment celles concernant le travail forcé) et Total va assez loin dans la reconnaissance du caractère problématique de la situation. Les réponses sont souvent convaincantes, même si quelques phrases aux accents très humanistes font sourire, notamment cette belle déclaration : « Aussi longtemps que nous estimerons que nos activités dans le cadre du projet Yadana contribuent au bien-être des populations du Myanmar, nous nous engageons à maintenir notre présence dans le pays et à démontrer qu’elle y est plus bénéfique pour ses habitants que notre absence. »

Plus concrètement, Total met en avant ses investissements en faveur des populations riveraines du gazoduc. Entre 1995 et 2005, près de 13 millions de dollars ont été dépensés ainsi. Un tel montant est relativement important pour un pays où un salaire mensuel de 30 dollars est chose courante. Ces fonds ont principalement été affectés au développement économique (avec un programme de mico-crédit), à la santé (85 000 consultations médicales gratuites en 2005 et une division par quatre du taux de mortalité infantile entre 1997 et 2005) et à l'éducation (avec des effectifs scolaires qui ont plus que doublé entre les années 1995/1996 et 2005/2006) ${ }^{13}$. Ces chiffres ne concernent certes que la population de 44000 personnes qui vivent dans les alentours du gazoduc, soit une proportion très faible des 47 millions d’habitants du pays. Ils ne sauraient pour autant être tenus pour négligeables.

Total met par ailleurs en avant son engagement en faveur du respect des droits internationaux du travail. Le groupe reconnaît que la situation est loin d'être parfaite sur ce plan, mais il affirme faire ce qui est en pouvoir pour empêcher le travail forcé et rémunérer correctement ceux qui travaillent pour lui. Total souligne aussi que si le groupe se retirait, un autre investisseur prendrait sa place. Du reste, un projet similaire est en cours d'étude avec un consortium regroupant des entreprises coréennes et indiennes ${ }^{14}$.

Total a par ailleurs soumis ses actions à l'évaluation d'organisations et d'experts indépendants. Le groupe a ainsi invité des représentants de l’ONG étasunienne Collaborative for Development Action (CDA), une organisation financée par des grands organismes publics de coopération et dont l'un des objectifs est d'assister des entreprises qui interviennent dans des « environnements complexes ${ }^{15}$. Des membres de cette ONG se sont rendus sur le site du gazoduc à plusieurs reprises entre 2002 et 2005 et les conclusions des rapports qu'ils ont rédigés sont plutôt favorables à Total ${ }^{16}$. Les auteurs reconnaissent les effets significatifs de l'engagement du groupe en faveur des populations qui résident dans les environs du gazoduc

\footnotetext{
${ }^{12}$ http://birmanie.total.com/ ; http://burma.total.com/ et http://myanmar.total.com/. On notera que Total utilise les deux noms : Birmanie et Myanmar.

${ }^{13}$ source : http://birmanie.total.com/index.htm

${ }^{14}$ voir : www.earthrights.org/burmareports/another_yadana_the_shwe_natural_gas_pipeline_project_burmabangladesh-india.html

15 www.cdainc.com/cep/

${ }^{16}$ Pour avoir accès aux 4 rapports voir : http://www.cdainc.com/publications/cep_field_visits.php
} 
et avalisent les efforts faits pour lutter contre le travail forcé. Ils écrivent même que la présence de Total (et l'attention internationale qui l'accompagne) protège les populations riveraines du travail forcé (qui continue à être utilisé par l'armée dans le reste du pays). Ceci n’élimine certes pas la possibilité que le gazoduc (dont le chantier a été achevé en 1998, soit 4 ans avant la première visite des représentants de CDA) ait été construit avec un recours au travail forcé (certains passages du premier rapport de CDA laissent même entendre que cela a bien été le cas), mais cela valide la prétention de Total à faire son possible pour lutter contre cette forme d'esclavagisme.

Total a par ailleurs commandé en 2003 une évaluation à Bernard Kouchner (via sa société BK conseil $)^{17}$. Ses conclusions convergent avec celles de CDA à ceci près que l'actuel ministre des Affaires étrangères prend une position plus nettement favorable à Total. Certains arguments sont toutefois osés : Bernard Kouchner compare notamment l'engagement du groupe en faveur des populations riveraines du gazoduc à celui d'organisations humanitaires telles que Médecins du monde ou Médecins sans frontière ${ }^{18}$. Il rappelle que la position de ces organisations est qu'il vaut mieux aider ceux que l'on peut aider plutôt que de ne rien faire du tout. Bernard Kouchner semble toutefois oublier que, si l'objet principal des organisations humanitaires est bien d'aider ceux qui souffrent, ce n'est pas le cas pour Total. En tout état de cause, son rapport n'a pas fait taire les critiques. Il a même suscité des commentaires acerbes de la part de nombreuses $\mathrm{ONG}^{19}$.

\section{La géopolitique du gaz en Asie du Sud-est}

Ces débats peuvent être rapprochés deux grandes questions. La première renvoie à un dilemme auquel les sociétés qui exploitent des ressources naturelles sont souvent confrontées : exploiter des gisements qui leur assurent des revenus ou préserver leur image, dont la qualité est elle aussi source de revenus. Dans le cas de Total, l'image du groupe n'est semble-t-il pas suffisamment écornée par les contestations pour remettre en question la profitabilité des investissements réalisés en Birmanie. En tout cas, le groupe se porte bien : il est l'une des toutes premières capitalisations de la bourse de Paris et engrange des bénéfices records.

La deuxième question est d'ordre moral (et éventuellement juridique via la possibilité d'une sanction par les tribunaux) : doit-on investir dans un pays totalitaire ? Cela peut-il avoir des effets positifs comme le prétendent certains défenseurs du libre-échange ? Ces derniers insistent ainsi sur les retombées positives du gazoduc pour les populations riveraines (du moins depuis l'achèvement de sa construction). Le fait que Total soit une entreprise très connue n’est évidemment pas étranger à cela, à la fois parce que le groupe pétrolier est

\footnotetext{
${ }^{17}$ Voir BK Conseil, Relation d'un voyage et de la découverte d'une industrie muette (après l'arrestation de Aung San Suu Lyi), 29 septembre 2003.

${ }^{18}$ Organisations dont Bernard Kouchner est l'un des co-fondateurs (Médecins du monde étant issue d'une scission opérée par Bernard Kouchner lui-même au sein de Médecins sans frontière).

${ }^{19}$ Voir : Véronique Smée, « Rapport Kouchner : les ONG consternées », 23 décembre 2005, site www.novethic.fr. Voir aussi la revue de presse sur : www.birmanie.net/birma/11_presse_birmanie.html
} 
sensible aux pressions internationales et parce que le gouvernement birman veut lui-même soigner son image autour de ce qui est pour lui un projet vitrine.

Au demeurant, 15 ans après la signature de l'accord entre le gouvernement birman et le groupe pétrolier, la situation politique locale ne s'est pas beaucoup améliorée. Total répond à cela que sa présence ne constitue qu'un élément d'ouverture internationale du pays et qu'il faudrait que les investissements étrangers se multiplient pour que la situation change véritablement, multiplication que la position des Etats-Unis et de la société civile internationale empêche. Cet argument est aussi avancé par Thant Myint-U, historien birman et ancien haut fonctionnaire de l'ONU. Pour lui : « On a perdu une occasion au début des années 1990, lorsque les militaires ont tenté d'ouvrir et de réformer l'économie ; si on avait participé à cette ouverture, on serait en bien meilleure position aujourd'hui pour peser sur un changement démocratique. ${ }^{20}$

Certains vont plus loin, affirmant qu'au regard de l'enjeu que représente la croissance économique, la démocratisation peut être considérée comme une question secondaire. Les exemples de la Chine et, plus encore, de Singapour sont souvent cités à l'appui de cette thèse. Ces exemples conduisent même des intellectuels à affirmer qu'un régime autoritaire peut être favorable au développement économique. Ces positions ont toutefois été fortement contestées par le prix Nobel d'économie Amartya Sen ${ }^{21}$, et la Birmanie est un exemple de pays autoritaire en situation économique difficile.

Quoi qu'il en soit, l'argument le plus fort de Total est que ce n'est pas à une entreprise privée de condamner un pays : si les investissements en Birmanie et le commerce avec ce pays doivent être bannis ce n'est pas à Total d'en décider, c'est l'affaire des instances régulatrices internationales. A elles de prendre leur responsabilité et d'imposer à Total un retrait de Birmanie si elles le jugent nécessaire. En l'état actuel de l’ordre géopolitique international, Total respecte le droit.

Certes, la sphère des échanges économiques n’est pas totalement indépendante de la sphère géopolitique, ainsi qu'en témoignent les réticences de la France à l'égard d'une attitude plus ferme de l'Europe vis-à-vis de la Birmanie ${ }^{22}$. Et, comme l'avait souligné un rapport de l'Assemblée nationale, l'ambassadeur de France en Birmanie a été, de 1999 à 2003, un ancien directeur des affaires internationales d'Elf, Bernard Amaudric du Chaffaut ${ }^{23}$. Il n'empêche : c'est aux responsables politiques de prendre position. Or, sur ce point, la situation est plus complexe que ce que veulent bien dire les ONG les plus critiques à l'égard de Total. En effet, le gazoduc mis en cause n'est pas à usage interne : à l'heure actuelle, plus de $90 \%$ du gaz extrait de Yadana est acheminé vers la Thaïlande pour y alimenter des centrales électriques. Si le gaz de Yadana est source de devises pour la Birmanie, c'est moins en raison de la présence de Total qu'en raison de la volonté de la Thaïlande et de sa compagnie nationale d'électricité

\footnotetext{
${ }^{20}$ Entretien réalisé par Sylvie Kauffmann pour Le Monde, publié sur le site Internet du quotidien le 19 octobre 2007.

${ }^{21}$ « Democracy as a universal value », Journal of Democracy, vol. 10, n 3, 1999, p. 3-17

${ }^{22} \mathrm{Il}$ est encore difficile de dire si les déclarations publiques de Nicolas Sarkozy sur Total seront suivies d'effets.

${ }^{23}$ Rapport déposé le 13 octobre 1999 par Marie-Hélène Aubert, Pierre Brana et Roland Blum sur « Le rôle des compagnies pétrolières dans la politique internationale et son impact social et environnemental ». Bernard Amaudric du Chaffaut est aujourd'hui ambassadeur en Azerbaïdjan, pays stratégique pour le gaz et le pétrole.
} 
d'acquérir ce gaz. La Thaïlande fait par ailleurs beaucoup pour maintenir son voisin birman dans le concert des nations considérées comme des partenaires commerciaux légitimes. Elle a notamment œuvré pour que la Birmanie devienne en 1997 membre de l’Association des nations du Sud-est asiatique (ASEAN).

En tout état de cause, la situation politique reste préoccupante en Birmanie. Avant les événements de 2007, l’absence d'évolution positive avait déjà conduit des organismes officiels tels que le Bureau international du travail à prendre le relais des ONG dans les actions juridiques à l'encontre du gouvernement birman. En outre, à la demande des EtatsUnis, le Conseil de sécurité de l’ONU avait décidé en septembre 2006 de mettre le « Myanmar » à son ordre du jour, « en raison du danger qu'il présente pour la sécurité internationale » ${ }^{24}$. Aujourd’hui, la pression diplomatique s'est accentuée et l'opinion internationale est, à nouveau, tournée vers la Birmanie.

Quel que puisse être l'effet de ces pressions, on ne peut qu'espérer un changement prochain. Si la Ligue nationale pour la démocratie présidée par Aung Suu Kyi prend le pouvoir, la situation risque de devenir difficile pour Total et la pérennité de son contrat pour l'exploitation du gisement de Yadana sera menacée (le contrat court jusqu'aux environs de 2030). La Nobel de la paix a en effet déclaré en 2000, lors de l’une de ses rares interventions : « Total a pris ses responsabilités en investissant massivement en Birmanie alors que d'autres décidaient de se retirer pour des raisons éthiques. La compagnie devra en assumer les conséquences. Le pays ne sera pas toujours gouverné par les dictateurs. » ${ }^{25}$

\footnotetext{
${ }^{24}$ http://www.un.org/apps/newsFr/storyF.asp?NewsID=13276\&Cr=Gambari\&Cr1=Myanmar

${ }^{25}$ dépêche AFP de mai 2000.
} 